\section{Prevalência e fatores associados a não inscrição para transplante renal}

\section{Prevalence and factors associated with non-enrollment for kidney transplant}

Prevalencia y factores asociados a la no inscripción para un trasplante renal
Francisca Maria Rodrigues dos Santos 1 Vera Lucia Mendes de Paula Pessoa 1 Raquel Sampaio Florêncio 1 Webertty Mayk Eufrásio de Figueirêdo 2 Paulo Henrique Pessoa Nobre 1 Tainá Veras de Sandes-Freitas 1,3

doi: 10.1590/0102-311X00043620

\section{Resumo}

Este estudo avaliou a prevalência e os fatores associados a não procura por transplante renal entre pacientes em diálise crônica na Região Metropolitana de Fortaleza, Ceará, Brasil. Foram excluídos os pacientes sem condições clínicas e aqueles em avaliação pré-transplante renal. Um questionário semiestruturado foi aplicado, incluindo opções para a pergunta "Qual o principal motivo pelo qual o senhor/senhora não está inscrito(a) para o transplante renal?”. A prevalência de pacientes considerados aptos, mas não inscritos e nem em avaliação pré-transplante renal foi de 50,7\%. As principais causas foram: receio de insucesso/perda do enxerto (32,5\%), dificuldade de transporte e acesso aos exames $(20,9 \%)$ e problemas pessoais ou familiares temporários $(13,7 \%)$. Em análise múltipla, as variáveis associadas a risco de receio do insucesso/perda do enxerto foram: sexo feminino $(O R=1,763$; IC95\%: 1,224-2,540) e doença renal dialitica (DRC 5-D) por hipertensão $(O R=1,732$; IC95\%: 1,178-2,547), tendo a renda mensal (salários mínimos) uma associação de proteção $(O R=$ 0,882; IC95\%: 0,785-0,991). O tempo em diálise (meses) foi um fator de risco para a dificuldade de transporte e acesso aos exames $(O R=1,004 ;$ IC95\%: 1,001-1,007) e o sexo feminino apresentou uma associação de proteção $(O R=$ 0,576; IC95\%: 0,368-0,901). Esses resultados mostram elevada prevalência de pacientes em diálise fora de lista para transplante renal. As principais causas são reflexo da desinformação e falta de acesso. Sexo feminino, baixa renda e DRC 5-D por hipertensão foram os fatores de risco para a não procura por transplante renal por receio de perda do enxerto, reflexo da carência de informações sobre a modalidade. Sexo masculino e maior tempo em diálise foram os fatores de risco para a dificuldade de acesso à terapia.

Insuficiência Renal Crônica; Transplante de Rim; Diálise
Correspondência

T. V. Sandes-Freitas

Universidade Federal do Ceará.

Rua Prof. Costa Mendes 1608, 4o andar, Fortaleza, CE 60416-200, Brasil.

taina.sandes@gmail.com

1 Universidade Estadual do Ceará, Fortaleza, Brasil.

2 Centro Universitário Inta - UNINTA, Sobral, Brasil.

3 Universidade Federal do Ceará, Fortaleza, Brasil. 


\section{Introdução}

Diálise e transplante renal são considerados terapias renais substitutivas (TRS), ou seja, tratamentos utilizados para pacientes com doença renal crônica estádio 5 dialítica (DRC 5-D ou terminal). Há fortes evidências demonstrando que o transplante renal é a TRS de escolha para pacientes com DRC 5-D 1,2,3. Mesmo com os notáveis avanços nas terapias dialíticas ocorridas na década de 1990 e nos anos 2000, o transplante renal continua proporcionando melhores resultados 3 . Além de reduzir a mortalidade, o transplante renal está associado à redução do risco de eventos cardiovasculares e infecções, e propicia melhor qualidade de vida. O benefício do transplante renal quando comparado à diálise está demonstrado mesmo em pacientes idosos 4 , naqueles com múltiplas comorbidades 5 , em pacientes que estão há um longo período em diálise 6 e nos que recebem rins considerados não ideais ou de critérios expandidos 7,8 .

Além das vantagens clínicas, o transplante renal apresenta a médio e longo prazos um custo inferior à permanência em diálise 9 . Um estudo recente realizado no Rio de Janeiro, Brasil, demonstrou que, após um período de 2 a 3 anos, o transplante renal resulta em uma economia de até R\$82.309,04 (já considerados os fármacos e tecnologias dialíticas atuais) 10.

De acordo com as Diretrizes Clínicas para o Cuidado ao Paciente com Doença Renal Crônica - DRC no Sistema Único de Saúde 11, publicadas em 2014, o transplante renal deve ser apresentado ao paciente ou ao seu representante legal dentro de 90 dias após o início da diálise. A diretriz recomenda ainda que o serviço de diálise deve referenciar o paciente a um serviço de transplante, encaminhando um relatório médico atualizado, além de se comprometer a enviar trimestralmente amostras de soro e informações sobre a situação clínica do paciente, bem como a ocorrência de eventos, como gestação, transfusão e óbito 11.

Nesse mesmo ano de 2014, foi publicada pelo Ministério da Saúde brasileiro a Portaria no 389 12, que "define os critérios para a organização da linha de cuidado da Pessoa com Doença Renal Crônica (DRC) e institui incentivo financeiro de custeio destinado ao cuidado ambulatorial pré-dialítico". Dentre os indicadores de qualidade estabelecidos pela Portaria, $80 \%$ dos pacientes aptos e com mais de seis meses de tratamento deveriam estar inscritos para o transplante renal 12.

Apesar dessas recomendações, dados do Censo Brasileiro de Diálise (CBD) da Sociedade Brasileira de Nefrologia (SBN) (http://www.censo-sbn.org.br/censosAnteriores, acessado em 08/Mar/2020) e do Registro Brasileiro de Transplantes (RBT) da Associação Brasileira de Transplante de Órgãos (ABTO) 13 mostram que, de um total estimado de 133.464 indivíduos em diálise no Brasil, apenas 29.545 (22,1\%) estavam inscritos para transplante renal em 2018. Não há evidências que embasem a utilização de um percentual mínimo desejado de pacientes inscritos em lista de espera dentre aqueles em diálise crônica. Esse percentual depende do perfil demográfico e clínico dos pacientes. No entanto, o estudo internacional Dialysis Outcomes and Practice Patterns Study (DOPPS) reportou percentuais de até 55\% 14.

Ressalte-se que o Brasil tem o segundo maior programa de transplante renal do mundo, realizando atualmente cerca de 6 mil transplante renal por ano. Quando considerados apenas os programas públicos de transplante, o Brasil passa a ser o primeiro do mundo, com mais de $90 \%$ dos transplante renal financiados pelo Sistema Único de Saúde (SUS) 13,15.

O Ceará tem uma situação bastante peculiar nesse cenário. Conta com uma Central Estadual de Transplantes (CET) com boa performance, que efetivou 22,8 doadores por milhão de população em 2018 - o que the conferiu o quarto lugar entre os estados brasileiros naquele ano - e um programa de transplante renal eficiente, realizando cerca de 300 transplante renal por ano. Isso resulta em um tempo de espera em lista abaixo da média nacional (que é de 50 a 60 meses) 16,17 e incentivo para a migração para a lista local de pacientes oriundos de estados vizinhos. Apesar desse eficiente programa de captação e transplante, em 2018 havia apenas 696 pacientes em lista de espera, o que representa 13,2\% dos 5.256 pacientes em diálise crônica no estado, um percentual bastante abaixo dos centros europeus desenvolvidos e também abaixo da média nacional (http://www.censo-sbn.org.br/censos Anteriores, acessado em 08/Mar/2020) 13.

Com o intuito de melhor compreender esse cenário, este estudo objetivou investigar os motivos pelos quais pacientes clinicamente aptos não estão inscritos para o transplante renal em nosso meio. Uma vez identificados esses motivos, as variáveis a eles associadas foram exploradas. 


\section{Métodos}

\section{Delineamento, amostra e local do estudo}

Estudo observacional transversal, predominantemente quantitativo e com elementos de natureza qualitativa, incluindo pacientes adultos (> 18 anos) prevalentes em programa de hemodiálise crônica há mais de três meses em clínicas de diálise localizadas na Região Metropolitana de Fortaleza, que abrange 19 municípios e cerca de 4 milhões de habitantes (Instituto de Pesquisa e Estratégia Econômica do Ceará. https://www.ipece.ce.gov.br/estatistica-e-geografia/, acessado em 24/Jun/2020). Foram excluídos: pacientes regularmente inscritos para transplante renal ou em avaliação ambulatorial prétransplante; aqueles considerados inaptos para transplante renal pela equipe clínica do serviço de diálise; impossibilidade cognitiva para responder ao questionário e ausência de acompanhante/cuidador que pudesse auxiliar na entrevista; os ausentes no dia da visita do entrevistador à clínica de diálise.

\section{Procedimentos dos estudo}

As entrevistas e coleta de dados foram realizadas entre agosto de 2018 e maio de 2019 por meio de um formulário semiestruturado, contendo opções para a pergunta "Qual o principal motivo pelo qual o senhor/senhora não está inscrito(a) para o transplante renal?”. As opções foram as que seguem: (i) receio de insucesso/perda do enxerto ("medo de não dar certo/medo de perder o rim"); (ii) receio de complicações clínicas e cirúrgicas ("medo da cirurgia e de ter problemas depois do transplante/ficar internado"); (iii) experiência negativa em transplante prévio (“já transplantei antes e não quero mais passar pelas mesmas coisas"); (iv) problemas pessoais ou familiares temporários (problemas pessoais ou na família no momento"); (v) dificuldade de transporte e acesso aos exames ("dificuldade para ir às consultas ou para fazer os exames do pré-transplante ou após o transplante"); (vi) ausência de informações sobre transplante renal ("nunca fui informado(a) sobre transplante"); (vii) paciente foi desencorajado por um profissional de saúde ("fui desencorajado(a) por um médico, enfermeiro ou alguém da área"); (viii) receio de perder o benefício previdenciário ("medo de perder benefício do Instituto Nacional do Seguro Social - INSS)”; (ix) outra opção não especificada acima.

Os pacientes que responderam "outra opção não especificada acima" foram convidados a falar livremente sobre estes motivos por meio de entrevistas gravadas, e as respostas foram analisadas por uma equipe composta por três profissionais independentes e classificadas em categorias.

Com o intuito de minimizar vieses, as entrevistas foram realizadas por apenas um profissional, previamente treinado.

Os dados sociodemográficos (sexo, idade, raça, escolaridade, renda, número de pessoas vivendo na mesma casa, religião, convênio/seguro de saúde) foram obtidos com os próprios pacientes, e os dados clínicos (etiologia da DRC 5-D, tempo em diálise, transplante prévio) foram obtidos dos prontuários multiprofissionais das clínicas de diálise.

\section{Análise dos dados}

As variáveis qualitativas foram expressas como frequências simples e porcentuais, e comparadas usando-se o teste de qui-quadrado ou Fisher. As variáveis numéricas foram expressas como média $(\mathrm{m})$, desvio padrão (DP), medianas (med) e intervalo interquartil (IIQ) no formato que segue: $\mathrm{m} \pm \mathrm{DP} / \mathrm{med}$ (IIQ). A análise univariada foi realizada por regressão logística binária, utilizando-se o cálculo da odds ratio (OR), com seu respectivo intervalo de 95\% de confiança (IC95\%). As variáveis cujo valor de p foi inferior a 0,15 na análise univariada foram incluídas no modelo múltiplo, por regressão logística binária, método stepwise. As variáveis com valor de p inferior a 0,05 foram consideradas significantes. As análises foram realizadas usando-se o software estatístico SPSS versão 24 (https://www.ibm.com/). 


\section{Requisitos éticos}

Este estudo seguiu todos os preceitos éticos regulamentados pela Resolução no 466/2012 do Conselho Nacional de Saúde e foi aprovado pelo Comitê de Ética em Pesquisa da Universidade Estadual do Ceará (UECE; protocolo no 2.780.656). Todos os participantes expressaram seu aceite assinando o Termo de Consentimento Livre e Esclarecido.

\section{Resultados}

\section{Amostra}

Das 26 clínicas de diálise instaladas no Ceará no período do estudo, 14 pertencem à Região Metropolitana de Fortaleza e 11 participaram da pesquisa. Dentre os 1.829 pacientes adultos prevalentes em diálise, 1.439 estavam em diálise há mais de três meses e foram considerados aptos pela equipe clínica. Desses, 731 (50,7\%) não estavam inscritos para transplante renal, nem em avaliação pré-transplante para a inscrição em lista. Trinta e cinco pacientes estavam ausentes nos dias das entrevistas na clínica e 127 se recusam participar da pesquisa, totalizando 569 pacientes entrevistados (Figura 1).

\section{Figura 1}

Definição da amostra de pacientes não inscritos para transplante renal na Região Metropolitana de Fortaleza, Ceará, Brasil, em 2019.

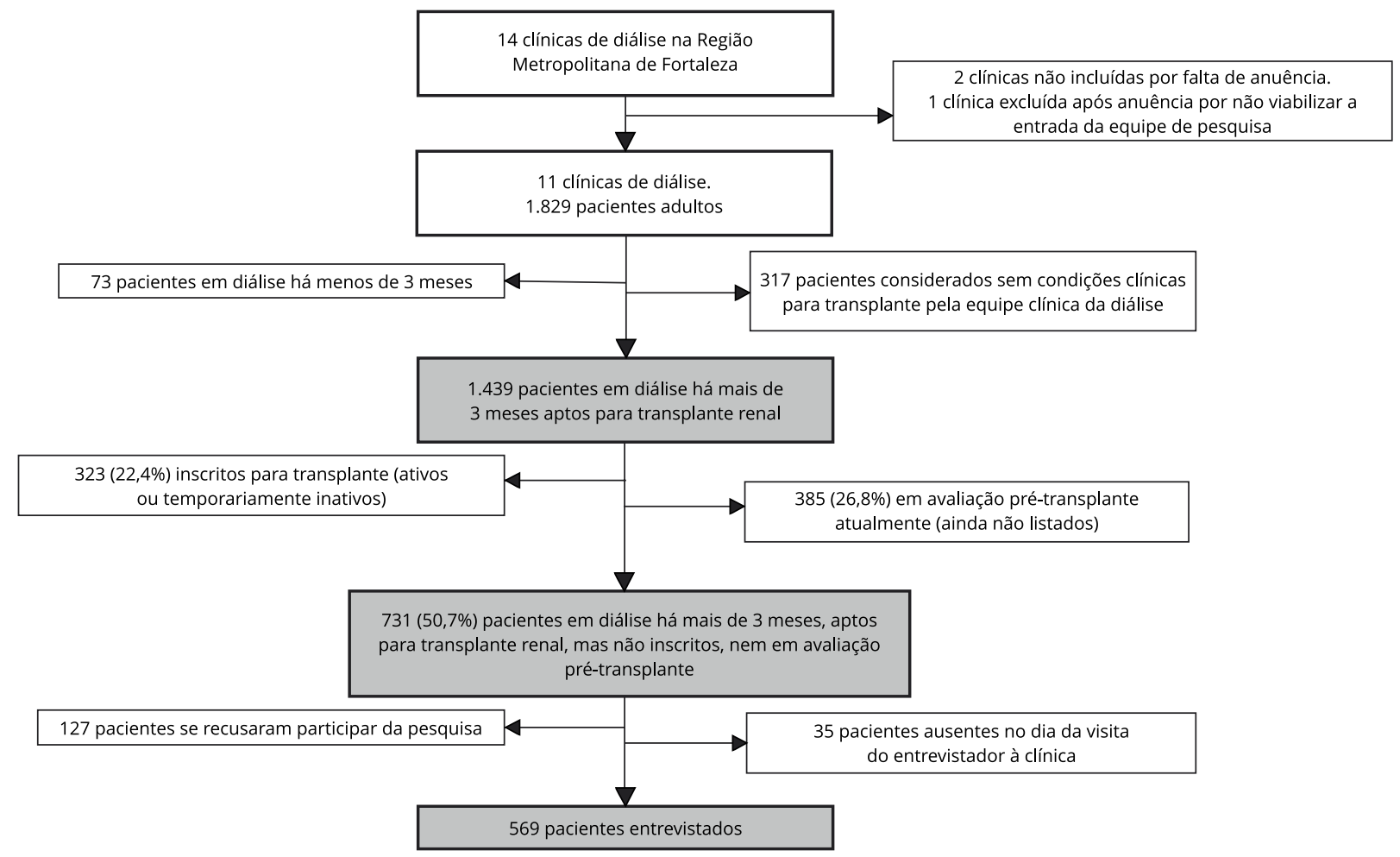




\section{Demografia}

Os pacientes eram predominantemente do sexo masculino (61,3\%), pardos (73,8\%), com idade média de 52,6 \pm 12,8 anos. A maioria deles (52\%) tinha o Ensino Fundamental completo ou incompleto e a renda média familiar de 2,1 \pm 3,0 salários mínimos, sendo 55,2\% casados ou amasiados, $66,4 \%$ católicos e com uma média de 2,1 \pm 1,5 pessoas residindo no mesmo domicílio. Quarenta e nove pacientes $(8,6 \%)$ possuíam convênio médico ou seguro de saúde privado. Diabetes foi a principal causa de DRC 5-D entre os entrevistados (34,6\%), seguida por hipertensão arterial (29\%) e etiologia não esclarecida (11,8\%). A mediana do tempo em diálise no momento da entrevista foi de 44 meses (IIQ 20-86,5 meses) e 16 pacientes $(2,8 \%)$ haviam sido submetidos a um transplante renal prévio (Tabela 1 ).

\section{Motivos para não buscar o transplantes como alternativa de tratamento à diálise}

A Figura 2 ilustra todas as causas referidas pelos pacientes como principais para não estarem inscritos para transplante renal. As três principais causas foram: receio do insucesso/perda do enxerto (185 pacientes; 32,5\%), dificuldade de transporte e acesso aos exames $(119 ; 20,9 \%)$ e problemas pessoais ou familiares temporários $(78 ; 13,7 \%)$.

\section{Variáveis associadas ao "receio do insucesso/perda do enxerto" e "dificuldade de transporte e acesso aos exames"}

Dentre os três principais motivos para a não inscrição para transplante renal, foram investigados os fatores associados às condições passíveis de intervenção pelos sistemas de saúde, apoio educacional e/ou assistência social. As Tabelas 2 e 3 descrevem esses resultados com detalhes.

As variáveis independentemente associadas a risco para "receio do insucesso/perda do enxerto" foram: o sexo feminino (OR = 1,763; IC95\%: 1,224-2,540; $\mathrm{p}=$ 0,002) e DRC 5-D por hipertensão arterial (OR = 1,732; IC95\%: 1,178-2,547; $\mathrm{p}=0,005)$, tendo a renda mensal em salários mínimos uma associação de proteção (OR = 0,882; IC95\%: 0,785-0,991; p = 0,035).

A única variável de risco para "dificuldade de transporte e acesso aos exames" foi o tempo em diálise em meses (OR = 1,004; IC95\%: 1,001-1,007; $\mathrm{p}=0,009)$. A variável sexo feminino apresentou uma associação de proteção (OR = 0,576; IC95\%: 0,368-0,901; p =0,016).

\section{Discussão}

Este estudo confirmou os dados dos censos e registros nacionais e internacionais quanto ao baixo percentual de pacientes em diálise crônica inscritos para o transplante renal em nosso meio $(17,7 \%)$. Dentre as principais causas, destacamos o receio de insucesso/perda do enxerto e as dificuldades de transporte e acesso aos exames complementares.

Ressalte-se que a amostra deste estudo foi constituída predominantemente por homens pardos, de meia idade, com baixo nível de instrução, renda mensal inferior a três salários mínimos, morando com poucas pessoas e sem convênio ou seguro de saúde suplementar. Esse cenário sociodemográfico provavelmente implica um ambiente de vulnerabilidade econômica e reduzida rede de proteção social, o que pode justificar os achados do estudo. Digno de nota, esse panorama é similar ao descrito pelo CBD da SBN para as demais diálises do país (http://www.censo-sbn.org.br/censosAnteriores, acessado em 08/Mar/2020).

Além disso, e também em linha com o CBD da SBN, a maioria dos pacientes da amostra apresentava uma doença sistêmica como causa da DRC 5-D (hipertensão arterial ou diabetes) (http://www. censo-sbn.org.br/censosAnteriores, acessado em 08/Mar/2020). De fato, é sabido que o percentual de pacientes com doença renal por doenças sistêmicas é menor em pacientes em lista de espera para o transplante ou transplantados, visto que uma parte deles não é considerada apta durante a avaliação clínica para o transplante 18,19. Entretanto, foram excluídos deste estudo os pacientes já considerados inaptos pela equipe assistencial da clínica de diálise e, portanto, seria esperado que os demais fossem avaliados por equipe especializada em transplante renal. 
Tabela 1

Características clínicas e sociodemográficas dos pacientes portadores de doença renal crônica estádio 5 dialítica (DRC 5-D) em hemodiálise e não inscritos para transplante renal na Região Metropolitana de Fortaleza, Ceará, Brasil, em $2019(n=569)$.

\begin{tabular}{|c|c|}
\hline Variáveis & Resultado * \\
\hline \multicolumn{2}{|l|}{ Sexo } \\
\hline Masculino & $349(61,3)$ \\
\hline Feminino & $220(38,7)$ \\
\hline Idade (anos) & $52,6 \pm 12,8 / 55(44-62)$ \\
\hline \multicolumn{2}{|l|}{ Raça autodeclarada } \\
\hline Parda & $420(73,8)$ \\
\hline Branca & $99(17,4)$ \\
\hline Preta & $50(8,8)$ \\
\hline \multicolumn{2}{|l|}{ Escolaridade } \\
\hline Analfabeto & $66(11,6)$ \\
\hline Ensino Fundamental ** & $296(52,0)$ \\
\hline Ensino Médio ** & $164(28,8)$ \\
\hline Ensino Superior ** & $43(7,6)$ \\
\hline Renda mensal familiar (salários mínimos) & $2,1 \pm 3,0 / 1(1-2)$ \\
\hline \multicolumn{2}{|l|}{ Estado civil } \\
\hline Solteiro(a) & $95(16,7)$ \\
\hline Casado(a) $* * *$ & $314(55,2)$ \\
\hline Separado(a)/Divorciado(a) & $109(19,2)$ \\
\hline Viúvo(a) & $51(9,0)$ \\
\hline Número de pessoas morando com o(a) paciente na mesma casa & $2,1 \pm 1,5 / 2(1-3)$ \\
\hline \multicolumn{2}{|l|}{ Convênio médico/Seguro de saúde privado } \\
\hline $\operatorname{Sim}$ & $49(8,6)$ \\
\hline Não & $520(91,4)$ \\
\hline \multicolumn{2}{|l|}{ Religião } \\
\hline Católico(a) & $378(66,4)$ \\
\hline Evangélico(a) & $170(29,9)$ \\
\hline Outra & $21(3,7)$ \\
\hline \multicolumn{2}{|l|}{ Etiologia da DRC 5-D } \\
\hline Diabetes mellitus & $197(34,6)$ \\
\hline Hipertensão & $165(29,0)$ \\
\hline Desconhecida & $67(11,8)$ \\
\hline Glomerulonefrite crônica & $49(8,6)$ \\
\hline Doença policística & $29(5,1)$ \\
\hline Uropatia & $25(4,4)$ \\
\hline Outra & $37(6,5)$ \\
\hline Tempo em diálise (meses) & $64,3 \pm 64,7 / 44(20-86,5)$ \\
\hline \multicolumn{2}{|l|}{ Transplante renal prévio } \\
\hline Sim & $16(2,8)$ \\
\hline Não & $553(97,2)$ \\
\hline
\end{tabular}

* Variáveis categóricas expressas como frequências e percentuais; variáveis numéricas expressas como média \pm desvio padrão/mediana (intervalo interquartil);

** Completo ou incompleto;

*** Incluindo casamento, união estável civil e união estável sem formalização. 


\section{Figura 2}

Motivos para a não inscrição para transplante renal entre pacientes em diálise crônica na Região Metropolitana de Fortaleza, Ceará, Brasil, em 2019.

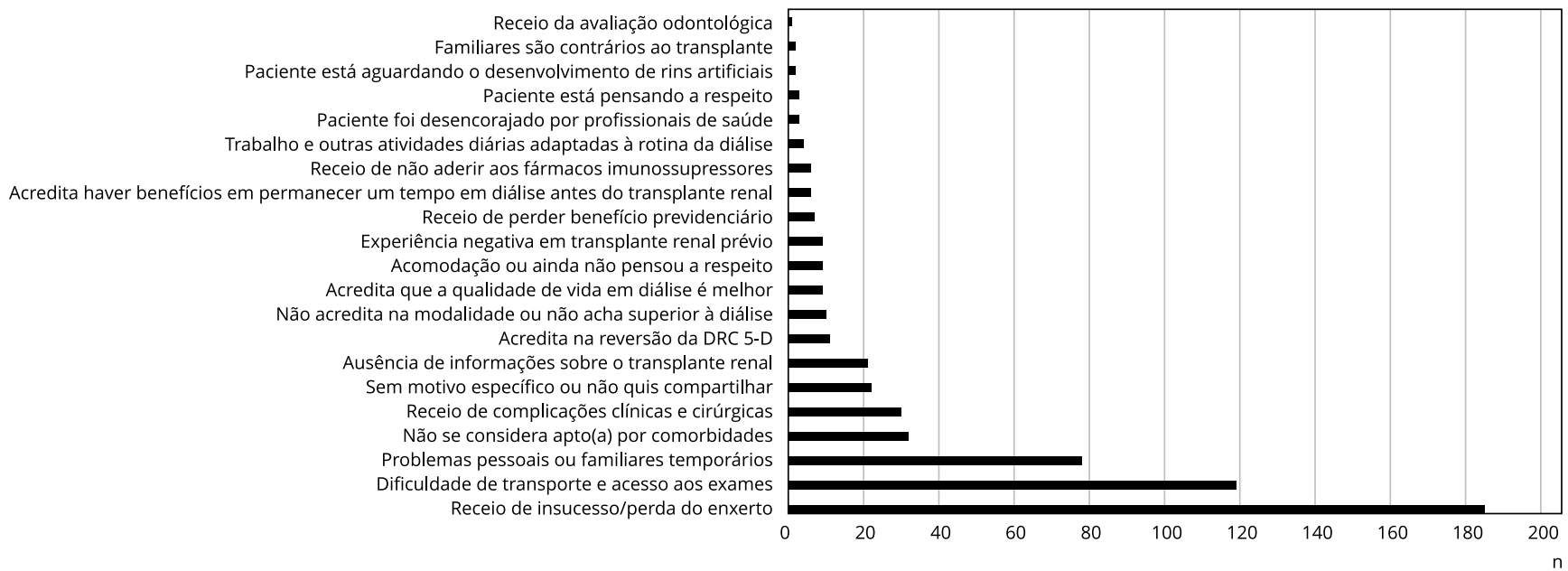

DRC 5-D: doença renal crônica estádio 5 dialítica.

\section{Tabela 2}

Variáveis associadas ao "receio do insucesso/perda do enxerto" em pacientes portadores de doença renal crônica estádio 5 dialítica (DRC 5-D) em hemodiálise e não inscritos para transplante renal na Região Metropolitana de Fortaleza, Ceará, Brasil, em 2019 ( $n=569$ ).

\begin{tabular}{|c|c|c|c|}
\hline \multirow[t]{2}{*}{ Variáveis independentes } & \multicolumn{3}{|c|}{ Análise univarida * } \\
\hline & OR & IC95\% & Valor de $p$ \\
\hline Sexo: feminino & 1,732 & $1,212-2,476$ & 0,003 \\
\hline Idade (anos) & 0,986 & 0,999-1,003 & 0,111 \\
\hline Raça: parda/preta (vs. branco) & 0,770 & $0,490-1,209$ & 0,257 \\
\hline Escolaridade: analfabeto/Ensino Fundamental (vs. Ensino Médio/Ensino Superior) ** & 0,752 & $0,656-1,357$ & 0,752 \\
\hline Renda (salários mínimos) & 0,876 & $0,777-0,988$ & 0,030 \\
\hline Estado civil: casado(a) (vs. solteiro(a)/separado(a)/viúvo(a)) & 1,099 & $0,772-1,565$ & 0,601 \\
\hline Número de pessoas morando com o paciente na mesma casa & 0,985 & $0,875-1,110$ & 0,808 \\
\hline Religião: cristão (vs. outras) & 2,096 & $0,695-6,320$ & 0,189 \\
\hline Etiologia da DRC 5-D: hipertensão (vs. as demais) & 1,657 & $1,136-2,417$ & 0,009 \\
\hline Tempo em diálise (meses) & 0,999 & $0,997-1,002$ & 0,717 \\
\hline Transplante prévio & 0,685 & $0,218-2,154$ & 0,518 \\
\hline Convênio médico/Seguro de saúde privado & 1,007 & $0,539-1,881$ & 0,983 \\
\hline \multirow[t]{2}{*}{ Variáveis independentes } & \multicolumn{3}{|c|}{ Análise múltipla * } \\
\hline & OR & IC95\% & Valor de $p$ \\
\hline Sexo: feminino & 1,763 & $1,224-2,540$ & 0,002 \\
\hline Renda (salários mínimos) & 0,882 & $0,785-0,991$ & 0,035 \\
\hline Etiologia da DRC 5-D: hipertensão (vs. as demais) & 1,732 & $1,178-2,547$ & 0,005 \\
\hline
\end{tabular}

IC95\%: intervalo de 95\% de confiança; OR: odds ratio.

* Análise por regressão logística binária. Entraram no modelo múltiplo as variáveis cujo valor de $\mathrm{p}<0,15$ na análise univariada;

** Completo ou incompleto. 
Tabela 3

Variáveis associadas à "dificuldade de transporte e acesso aos exames" em pacientes portadores de doença renal crônica estádio 5 dialítica (DRC 5-D) em hemodiálise e não inscritos para transplante renal na Região Metropolitana de Fortaleza, Ceará, Brasil, em 2019 (n = 569).

\begin{tabular}{|c|c|c|c|}
\hline \multirow[t]{2}{*}{ Variáveis independentes } & \multicolumn{3}{|c|}{ Análise univariada * } \\
\hline & OR & IC95\% & Valor de $p$ \\
\hline Sexo: feminino & 0,658 & $0,628-1,013$ & 0,057 \\
\hline Idade (anos) & 1,001 & $0,985-1,017$ & 0,927 \\
\hline Raça: parda/preta (vs. branco) & 1,232 & $0,706-2,148$ & 0,463 \\
\hline Escolaridade: analfabeto/Ensino Fundamental (vs. Ensino Médio/Ensino Superior) ** & 1,013 & $0,666-1,543$ & 0,950 \\
\hline Renda (salários mínimos) & 0,925 & $0,820-1,043$ & 0,202 \\
\hline Estado civil: casado(a) (vs. solteiro(a)/separado(a)/viúvo(a)) & 0,752 & $0,502-1,127$ & 0,168 \\
\hline Número de pessoas morando com o paciente na mesma casa & 0,980 & $0,854-1,125$ & 0,776 \\
\hline Religião: cristão (vs. outras) & 0,649 & $0,246-1,712$ & 0,383 \\
\hline Etiologia da DRC 5-D: hipertensão (vs. as demais) & 0,787 & $0,496-1,246$ & 0,307 \\
\hline Tempo em diálise (meses) & 1,003 & $1,000-1,006$ & 0,025 \\
\hline Transplante prévio & 0,246 & $0,032-1,880$ & 0,176 \\
\hline Convênio médico/Seguro de saúde privado & 0,503 & $0,209-1,211$ & 0,125 \\
\hline \multirow[t]{2}{*}{ Variáveis independentes } & \multicolumn{3}{|c|}{ Análise múltipla * } \\
\hline & OR & IC95\% & Valor de p \\
\hline Sexo: feminino & 0,576 & $0,368-0,901$ & 0,016 \\
\hline Tempo em diálise (meses) & 1,004 & $1,001-1,007$ & 0,009 \\
\hline
\end{tabular}

IC95\%: intervalo de 95\% de confiança; OR: odds ratio.

* Análise por regressão logística binária. Entraram no modelo múltiplo as variáveis cujo valor de p $<0,15$ na análise univariada;

** Completo ou incompleto.

Não há dados publicados sobre as diferenças no perfil sociodemográfico de pacientes com DRC 5-D das capitais e regiões metropolitanas brasileiras comparados aos de pacientes residentes no interior dos estados. No entanto, considerando o contexto socioeconômico da Região Nordeste e as diferenças nos indicadores sociais entre Fortaleza e Região Metropolitana e as cidades do interior do Estado do Ceará 20, é provável que o percentual de pacientes em diálise crônica nas cidades do interior do estado não inscritos para o transplante renal seja ainda maior. Soma-se a isso o fato de que os centros de transplante atualmente ativos do estado estão localizados na capital, o que dificulta a logística para a realização de consulta e exames pré-transplante.

O principal motivo para os pacientes aptos não estarem inscritos foi o receio do insucesso do transplante ou perda do enxerto (32,5\%), provável reflexo da desinformação sobre os desfechos do transplante renal. É sabido que diversas condições podem impactar na sobrevida do enxerto renal e do paciente, algumas resultando em perda do enxerto e óbito precoce. Entretanto, de acordo com dados do RBT da ABTO, a sobrevida em um ano de um enxerto de doador falecido é de $84 \%$. Quando considerado o óbito como causa de perda do enxerto, os dados do RBT mostram sobrevida de 92\% em um ano para transplantes com doador falecido. Ressalte-se que esses dados do RBT representam a média nacional e não a experiência de centros de excelência, onde as sobrevidas do enxerto e do paciente costumam ser ainda melhores 17,21,22. Infelizmente, não há dados publicados sobre a mortalidade em lista de espera no Brasil. Evidências de estudos internacionais demonstram que, passados os primeiros 100-300 dias após o transplante renal, o risco de morrer é inferior à permanência em lista de espera 1,7, indicando que apesar do risco de perda do enxerto ou óbito esta terapia é superior à permanência em diálise e esta superioridade já é demonstrada nos primeiros meses após o transplante renal, quando superados os riscos perioperatórios. É importante ressaltar que pacientes em diálise são constantemente expostos a indivíduos que retornam à diálise após a perda do enxerto, seja precoce, seja após 
longos anos de um transplante renal bem-sucedido. Com frequência, tais pacientes encontram-se em condições clínicas piores quando comparadas ao início da diálise após a perda da função renal dos rins nativos 23. Por outro lado, pacientes em diálise habitualmente não têm acesso a pacientes com transplante renal bem-sucedido. Alguns centros de transplante têm relatado experiências positivas com pacientes transplantados renais com enxerto funcionante exercendo atividade de navigators, ou seja, indivíduos que auxiliam o potencial candidato a transplante renal durante todo o processo, incluindo esclarecimentos sobre a experiência de transplantar 24,25.

Ainda dentro dessa perspectiva de desinformação sobre os resultados do transplante renal, outras respostas chamaram atenção: 32 pacientes não se consideraram aptos para transplante renal em função de comorbidades e idade avançada; dez não acreditavam na superioridade do transplante renal quando comparado à diálise; e nove achavam que a qualidade de vida em diálise é melhor. No entanto, há evidências muito contundentes demonstrando que o transplante renal proporciona menor morbimortalidade quando comparado à diálise 3 e estes resultados independem da idade ${ }^{4} \mathrm{e}$ da presença de comorbidades 5 . Adicionalmente, diversos estudos indicam a superioridade na qualidade de vida dos pacientes transplantados quando comparados àqueles que permanecem em diálise 3.

Como mencionado anteriormente, o tempo em lista de espera para transplante renal no Estado do Ceará está abaixo das médias nacionais. Entretanto, o tempo total em diálise é elevado e isto ocorre porque, na maior parte do tempo em diálise, esses pacientes estão fora da lista de espera. Dados locais recentemente publicados mostraram que pacientes com DRC 5-D transplantados sob alocação regular permaneceram longos períodos em diálise fora de lista (31,2 \pm 29 meses, med $=21,5$ meses). Após listados, o tempo de espera foi de 7,9 $\pm 7,3$ meses (med $=4,8$ meses). Um grupo de pacientes transplantados sob urgência por falência de acesso vascular permaneceu, em média, 54,5 $\pm 52,5$ meses (med $=33,9$ meses) em diálise antes de todos os acessos se extinguirem. Os pacientes transplantados nessa situação apresentaram desfechos do transplante piores que os de pacientes transplantados sob alocação regular, incluindo um elevado percentual de perda do enxerto por trombose 17 . Seis pacientes entrevistados afirmaram não estar inscritos por acreditarem haver benefício de permanecer um tempo em diálise antes de transplantar, mais uma resposta que reflete desinformação sobre o transplante renal. Há evidências muito contundentes demonstrando que, quanto menor o tempo em diálise, melhores os resultados 26 .

A falta de informação é sabidamente uma importante barreira de acesso ao transplante, uma vez que embota o processo decisório do paciente quanto às modalidades de TRS disponíveis.

Assim como neste levantamento, um estudo americano demonstrou que, dentre outros fatores, mulheres, pacientes de baixa renda e portadores de DRC 5-D por hipertensão arterial estão sob maior risco de não receberem informações sobre o transplante renal 27. Não há evidências que expliquem a inequidade entre os sexos no acesso ao transplante, entretanto, é provável que as mulheres estejam sob maior vulnerabilidade social e psicológica, dificultando o acesso à informação 28. É compreensível que pacientes com doença renal por hipertensão arterial tenham menor acesso à lista por questões clínicas relativas ao acometimento de outros órgãos-alvo, como o coração. Entretanto, não há evidências prévias que apontem que sejam de risco para falta de acesso à lista por desinformação sobre a modalidade. Variáveis não exploradas neste estudo, como aquelas relacionadas ao autocuidado e letramento em saúde, podem explicar esses achados.

Chama ainda atenção que 21 pacientes não procuraram o transplante renal porque não receberam informações sobre a modalidade em sua trajetória de doença renal em tratamento conservador e em diálise. Outros três foram desencorajados por algum profissional de saúde, o que sugere que o conhecimento destes profissionais sobre o transplante renal é deficitário.

$\mathrm{O}$ segundo maior motivo relatado foi a dificuldade de transporte para comparecer às consultas pré e pós-transplante, e de operacionalizar os exames complementares rotineiramente solicitados (119 pacientes, 20,9\%). Sete pacientes optaram por não se inscrever por receio de perder o benefício previdenciário concedido a pacientes com DRC 5-D, o que corrobora o diagnóstico de considerável vulnerabilidade social a que estes pacientes estão submetidos. A vulnerabilidade social é um conhecido fator de risco para o pior acesso aos serviços de saúde, gerando inequidade. Evidências demonstram que mesmo em países desenvolvidos a "privação social" é uma importante barreira de acesso ao transplante renal 29. Nesta amostra, as mulheres estiveram menos susceptíveis a essa barreira. Não há evidências disponíveis que expliquem de forma definitiva esse achado e é possível que isso esteja rela- 
cionado à maior disposição das mulheres em buscar soluções para o autocuidado 30. Por outro lado, quanto maior o tempo em diálise, maiores as dificuldades para acessar o centro e operacionalizar os exames. Igualmente, não há evidências que justifiquem esse achado. É importante, entretanto, lembrar que este é um estudo transversal e as análises não refletem causalidade.

Este trabalho tem limitações que devem ser apontadas: (a) é um estudo cuja população foi restrita a uma região, não sendo possível extrapolar essas informações para outras regiões do país; (b) por dificuldades logísticas de deslocamento da equipe de pesquisa não foram incluídos pacientes do interior do Ceará, cuja realidade social é distinta da Grande Fortaleza, o que pode implicar uma prevalência menor de pacientes inscritos para transplante renal e motivações diferentes, além disto, de acordo com os dados do RBT, não há centros de transplante renal ativos no interior do estado no momento, sendo necessário o deslocamento até a capital, o que também pode gerar dificuldades; (c) não foram incluídos pacientes em diálise peritoneal por dificuldades de acessá-los, os quais realizam seu tratamento no domicílio, entretanto, o acesso a esta diálise no nosso meio é bastante restrito e estima-se que represente menos de $5 \%$ do total de pacientes em diálise na região; (d) este estudo não foi desenhado com o objetivo primário de avaliar fatores de risco, portanto, estas análises inferenciais têm as limitações relativas ao desenho e à amostra.

Mesmo com essas limitações, este trabalho tem forças muito importantes. Apesar da disponibilidade dos censos nacionais, a apuração mais adequada do percentual de pacientes inscritos para transplante renal por região apenas pode ser realizada por meio de estudos desta natureza. Isso porque o paciente portador de DRC 5-D tem o direito de escolher o centro de transplante de sua preferência. Isso implica que alguns pacientes em diálise no Ceará contabilizados pelo CBD podem estar inscritos em outros estados do país. Da mesma forma, dentre os listados para transplante renal no Ceará há um número não desprezível de pacientes oriundos de outros estados.

Além disso, este é o primeiro estudo nacional que investigou os motivos para a não inscrição em lista de espera para transplante renal de pacientes prevalentes em diálise crônica, uma realidade que resulta em maior morbimortalidade e pior qualidade de vida aos pacientes, maior custo aos sistemas de saúde, além de predispor a falência de acessos vasculares após o longo período em diálise, resultando em piores resultados do transplante renal realizado sob urgência. Apesar disso, o transplante renal é uma possibilidade de reabilitar o indivíduo para a vida laboral, aumentando a geração de riqueza para a região.

Em conclusão, é elevada a prevalência de pacientes em diálise fora da lista para transplante renal. As principais causas para esse cenário concentram-se em dois aspectos: desinformação e dificuldade de acesso. Este estudo pode servir como uma fonte para a implantação de medidas para remediar esse cenário, como: ações sociais incluindo a visita de pacientes com transplante renal bem-sucedido em clínicas de diálise; contratação destes pacientes como navigators para auxiliar em todo o processo; ações educativas voltadas para pacientes e profissionais de saúde esclarecendo sobre os resultados do transplante; ações de assistência social para garantir o acesso dos pacientes a transporte e realização de exames complementares; e, por fim, a melhora na comunicação entre as unidades de diálise e o centro de transplante, dirimindo dúvidas, evitando redundâncias em exames complementares, ajustando as visitas ao centro de transplante nos dias de diálise e minimizando o número de visitas necessárias ao centro para inscrição e ativação em lista. 


\section{Colaboradores}

F. M. R. Santos participou da concepção e planejamento do estudo, coleta, tabulação, análise e interpretação dos dados, redação e revisão do manuscrito. V. L. M. P. Pessoa colaborou na coleta, tabulação, análise e interpretação dos dados e redação do manuscrito. R. S. Florêncio contribuiu na análise e interpretação dos dados e revisão do manuscrito. W. M. E. Figueirêdo e P. H. P. Nobre colaboraram na coleta e tabulação dos dados e revisão do manuscrito. T. V. Sandes-Freitas participou da concepção e planejamento do estudo, análise e interpretação dos dados e redação do manuscrito.

\section{Informações adicionais}

ORCID: Francisca Maria Rodrigues dos Santos (0000-0002-5599-858X); Vera Lucia Mendes de Paula Pessoa (0000-0002-8158-7071); Raquel Sampaio Florêncio (0000-0003-3119-7187); Webertty Mayk Eufrásio de Figueirêdo (0000-0002-8848653X); Paulo Henrique Pessoa Nobre (0000-00027693-8607); Tainá Veras de Sandes-Freitas (00000002-4435-0614).

\section{Agradecimentos}

Agradecemos a todos os responsáveis técnicos, enfermeiros, assistentes sociais e demais membros do staff das unidades de diálise incluídas neste estudo, os quais viabilizaram de forma cordial a visita da equipe e o acesso aos pacientes.

\section{Referências}

1. Wolfe RA, Ashby VB, Milford EL, Ojo AO, Ettenger RE, Agodoa LY, et al. Comparison of mortality in all patients on dialysis, patients on dialysis awaiting transplantation, and recipients of a first cadaveric transplant. N Engl J Med 1999; 341:1725-30.

2. Oniscu GC, Brown H, Forsythe JL. Impact of cadaveric renal transplantation on survival in patients listed for transplantation. J Am Soc Nephrol 2005; 16:1859-65.

3. Tonelli M, Wiebe N, Knoll G, Bello A, Browne S, Jadhav D, et al. Systematic review: kidney transplantation compared with dialysis in clinically relevant outcomes. Am J Transplant 2011; 11:2093-109.

4. Rao PS, Merion RM, Ashby VB, Port FK, Wolfe RA, Kayler LK. Renal transplantation in elderly patients older than 70 years of age: results from the Scientific Registry of Transplant Recipients. Transplantation 2007; 83:1069-74.

5. Sorensen VR, Heaf J, Wehberg S, Sorensen SS. Survival benefit in renal transplantation despite high comorbidity. Transplantation 2016; 100:2160-7.

6. Rose C, Gill J, Gill JS. Association of kidney transplantation with survival in patients with long dialysis exposure. Clin J Am Soc Nephrol 2017; 12:2024-31.

7. Ojo AO, Hanson JA, Meier-Kriesche H, Okechukwu CN, Wolfe RS, Leichtman AB, et al. Survival in recipients of marginal cadaveric donor kidneys compared with other recipients and wait-listed transplant candidates. J Am Soc Nephrol 2001; 12:589-97.

8. Massie AB, Luo X, Chow EK, Alejo JL, Desai NM, Segev DL. Survival benefit of primary deceased donor transplantation with high-KDPI kidneys. Am J Transplant 2014; 14:2310-6.

9. Arredondo A, Rangel R, Icaza E. Cost-effectiveness of interventions for end-stage renal disease. Rev Saúde Pública 1998; 32:556-65.

10. Silva SB, Caulliraux HM, Araújo CA, Rocha E. Uma comparação dos custos do transplante renal em relação às diálises no Brasil. Cad Saúde Pública 2016; 32:e00013515.

11. Departamento de Atenção Especializada e Temática, Secretaria de Atenção à Saúde, Ministério da Saúde. Diretrizes clínicas para o cuidado ao paciente com doença renal crônica - DRC no Sistema Único de Saúde. Brasília: Ministério da Saúde; 2014.

12. Ministério da Saúde. Portaria no 383, de 13 de março de 2014. Define os critérios para a organização da linha de cuidado da pessoa com Doença Renal Crônica (DRC) e institui incentivo financeiro de custeio destinado ao cuidado ambulatorial pré-dialítico. Diário Oficial da União 2014; 14 mar.

13. Associação Brasileira de Transplantes de Órgãos. Registro Brasileiro de Transplantes: dimensionamento dos transplantes no brasil e em cada estado (2011-2018). São Paulo: Associação Brasileira de Transplantes de Órgãos; 2019. 
14. Satayathum S, Pisoni RL, McCullough KP, Merion RM, Wikström B, Levin N, et al. Kidney transplantation and wait-listing rates from the international Dialysis Outcomes and Practice Patterns Study (DOPPS). Kidney Int 2005; 68:330-7.

15. Medina-Pestana JO, Galante NZ, Tedesco-Silva Jr. H, Harada KM, Garcia VD, Abbud-Filho $\mathrm{M}$, et al. $\mathrm{O}$ contexto do transplante renal no Brasil e sua disparidade geográfica. J Bras Nefrol 2011; 33:472-84.

16. Medina-Pestana JO. Organization of a highvolume kidney transplant program: the "assembly line" approach. Transplantation 2006; 81:1510-20.

17. Sandes-Freitas TV, Oliveira JGR, Dantas GLA, Mesquita LL, Almeida ERB, Esmeraldo RM. Clinical characteristics and outcomes of kidney transplantation under urgency priority condition. Nephron 2019; 142:98-105.

18. Machado EL, Gomes IC, Acurcio FA, César CC, Almeida MC, Cherchiglia ML. Fatores associados ao tempo de espera e ao acesso ao transplante renal em Belo Horizonte, Minas Gerais, Brasil. Cad Saúde Pública 2012; 28:2315-26.

19. Oniscu GC, Schalkwijk AA, Johnson RJ, Brown $\mathrm{H}$, Forsythe JL. Equity of access to renal transplant waiting list and renal transplantation in Scotland: cohort study. BMJ 2003; 327:1261-5.

20. Instituto de Pesquisa Econômica Aplicada. Atlas do desenvolvimento humano no Brasil. http://www.atlasbrasil.org.br/2013/pt/home/ (acessado em 24/Jun/2020).

21. Bicalho PR, Requião-Moura LR, Arruda EF, Chinen R, Mello L, Bertocchi APF, et al. Longterm outcomes among kidney transplant recipients and after graft failure: a single-center cohort study in brazil. Biomed Res Int 2019; 2019:7105084.

22. Harada KM, Mandia-Sampaio EL, Sandes-Freitas TV, Felipe CR, Park SI, Pinheiro-Machado PG, et al. Risk factors associated with graft loss and patient survival after kidney transplantation. Transplant Proc 2009; 41:3667-70.
23. Arias M, Escallada R, Francisco AL, Rodrigo E, Fernández-Fresnedo G, Setién MA, et al. Return to dialysis after renal transplantation. Which would be the best way? Kidney Int Suppl 2002; (80):85-8.

24. Sullivan C, Dolata J, Barnswell KV, Greenway K, Kamps CM, Marbury Q, et al. Experiences of kidney transplant recipients as patient navigators. Transplant Proc 2018;50:3346-50.

25. Sullivan C, Leon JB, Sayre SS, Marbury M, Ivers M, Pencak JA, et al. Impact of navigators on completion of steps in the kidney transplant process: a randomized, controlled trial. Clin J Am Soc Nephrol 2012; 7:1639-45.

26. Meier-Kriesche HU, Kaplan B. Waiting time on dialysis as the strongest modifiable risk factor for renal transplant outcomes: a paired donor kidney analysis. Transplantation 2002; 74:1377-81.

27. Kucirka LM, Grams ME, Balhara KS, Jaar BG, Segev DL. Disparities in provision of transplant information affect access to kidney transplantation. Am J Transplant 2012; 12:351-7.

28. Couchoud C, Bayat S, Villar E, Jacquelinet C, Ecochard R; REIN Registry. A new approach for measuring gender disparity in access to renal transplantation waiting lists. Transplantation 2012; 94:513-9.

29. Dudley CR, Johnson RJ, Thomas HL, Ravanan R, Ansell D. Factors that influence access to the national renal transplant waiting list. Transplantation 2009; 88:96-102.

30. Machin R, Couto MT, Silva GS, Schraiber LB, Gomes R, Figueiredo WS, et al. Concepções de gênero, masculinidade e cuidados em saúde: estudo com profissionais de saúde da atenção primária. Ciênc Saúde Colet 2011; 16:450312. 
Abstract

This study evaluated the prevalence and factors associated with lack of enrollment for kidney transplant among patients in chronic dialysis in Greater Metropolitan Fortaleza, Ceará, Brazil. The sample excluded patients with insufficient clinical status and those already in pre-kidney transplant evaluation. A semi-structured questionnaire was applied, including options for the question, "What is the main reason why you are not enrolled for kidney transplant?" Prevalence of patients considered fit but not enrolled or in pre- kidney transplant evaluation was $50.7 \%$. The main reasons were fear of failure/loss of grafting (32.5\%), difficulty with transportation or access to tests (20.9\%), and temporary personal or family problems (13.7\%). In the multivariate analysis, the variables associated with fear of failure or loss of graft were female sex (OR $=1.763$; 95\% CI: 1.2242.540) and end-stage renal disease (ESRD) due to hypertension ( $\mathrm{OR}=1.732$; 95\%CI: 1.178-2.547), while monthly income (number of minimum wages) showed a protective association ( $O R=0.882$; 95\%CI: 0.785-0.991). Time on dialysis (months) was a risk factor for difficulty with transportation and access to tests (OR = 1.004; 95\%CI: 1.001 1.007), and female sex showed a protective association (OR $=0.576$; 95\%CI: 0.368-0.901). These results show high prevalence of patients in dialysis not enrolled on the kidney transplant waitlist. The main causes were lack of information and lack of access. Female sex, low income, and ESRD due to hypertension were risk factors for lack of enrollment on the kidney transplant waitlist due to fear of loss of graft, resulting from lack of information on this treatment modality. Male sex and longer time on dialysis were risk factors for difficulty in access to kidney transplant.

Chronic Renal Insufficiency; Kidney

Transplantation; Dialysis

\section{Resumen}

Este estudio evalúo la prevalencia y los factores asociados a la no búsqueda de un trasplante renal entre pacientes con diálisis crónica en la Región Metropolitana de Fortaleza, Ceará, Brasil. Se excluyeron a pacientes sin condiciones clínicas $y$ aquellos en evaluación pre-trasplante renal. Se aplicó un cuestionario semiestructurado, incluyendo opciones a la pregunta "¿cuál es el principal motivo por el cual usted no está inscrito(a) para un trasplante renal?" La prevalencia de pacientes considerados aptos, pero no inscritos y ni en evaluación pre-trasplante renal fue de un 50,7\%. Las principales causas fueron: recelo al fracaso/pérdida del injerto (32,5\%), dificultad de transporte y acceso a los exámenes (20,9\%), así como problemas personales o familiares temporales (13,7\%). En el análisis múltiple, las variables asociadas al riesgo del recelo al fracaso/pérdida del injerto fueron de sexo femenino (OR = 1,763; IC95\%: 1,224-2,540) $y$ enfermedad renal dialítica (DRC 5-D) por hipertensión $(O R=1,732$; IC95\%: 1,178-2,547), teniendo la renta mensual (salarios mínimos) una asociación de protección (OR = 0,882; IC95\%: 0,785-0,991). El tiempo en diálisis (meses) fue un factor de riesgo para la dificultad en el transporte y acceso a los exámenes $(O R=1,004$; IC95\%: 1,001-1,007), y el sexo femenino tuvo una asociación de protección (OR = 0,576; IC95\%: 0,368$0,901)$. Estos resultados muestran la elevada prevalencia de pacientes en diálisis fuera de la lista para transplante renal. Las principales causas son reflejo de la desinformación y falta de acceso. Sexo femenino, baja renta y DRC 5-D por hipertensión fueron factores de riesgo para la no búsqueda de trasplante renal por recelo a la pérdida del injerto, reflejo de la carencia de información sobre la modalidad. Sexo masculino y mayor tiempo en diálisis fueron factores de riesgo para la dificultad de acceso a la terapia. izará la atención a las vulnerabilidades individuales desde la perspectiva de la salud integral.

Insuficiencia Renal Crónica; Trasplante de Riñón; Diálisis
Recebido em 11/Mar/2020

Versão final reapresentada em 15/Out/2020

Aprovado em 26/Out/2020 